\title{
Ballast flight under high-speed trains: wind tunnel full-scale
} experimental tests

\author{
Premoli, A. ${ }^{a}$, Rocchi, D. ${ }^{a}$, Schito, P. ${ }^{a}$, Somaschini, C. ${ }^{* a}$, Tomasini, G. $^{\mathrm{a}}$ \\ ${ }^{a}$ Politecnico di Milano, Department of Mechanical Engineering \\ Via la Masa 1, Milano, Italy. \\ *claudio.somaschini@polimi.it \\ Ph. 00390223998466
} (1) (1) (1) (1) 8

\begin{abstract}
The flying ballast phenomenon has become an important problem, in the last years, because of the development of high speed trains and the consequent increase of the speed up to $350 \mathrm{~km} / \mathrm{h}$. The problem is very complex since it is related to both railway infrastructure and train characteristics and since it involves mechanical and aerodynamic effects. The results of an experimental study carried out on the Italian high-speed railway and on a 1:1 real stretch of the railways in wind tunnel are presented in the paper. The study was aimed to analyze the effects of the height of the ballast level, the stone shape in the upper layer of the ballast and the compaction of the ballast bed on the problem. To this purpose a specific wind tunnel test rig was designed to reproduce in the wind tunnel a flow with the same average characteristics of the one measured on the real line, especially in the region close to the ballast and sleepers. Finally, starting from the results of these tests, possible countermeasures to ballast lifting on-set are proposed.
\end{abstract}

KEYWORDS: Flying ballast, train aerodynamics, high-speed railways, wind tunnel, full-scale experiments.

\section{INTRODUCTION}

The phenomenon of ballast-flying is one of the major problems caused by the increase in railway speed over $300 \mathrm{~km} / \mathrm{h}$ in terms of safety and early deterioration of both rolling stock and railway. 
Generally, the ballast lifting phenomenon can arise also at low speed, due to external agents as ice or other materials on the line (Jing et al., 2012 and Kaltenbach et al., 2008). On the other hand, the problem becomes extremely evident increasing the speed, when the ballast stones are lifted up due to the pressure and velocity field generated in the upper layer of ballast by the train. The consequences of this phenomenon are different: on the safety of the people working along railway lines, on the running safety of the trains themselves, and, finally, on the extra costs associated to both the rolling stock and the infrastructure maintenance (problem of ballast pitting, Quinn et al., 2010).

Furthermore, nowadays the issue of ballast-lifting is not regulated and limited by any international standard. For this reason, in the last years, within two European projects, Aerodynamic in Open Air (AOA within the DEUFRAKO project, 2006-2008) and Aerotrain (2008-2012), the main infrastructure managers and rolling stock constructors (SNCF, DB, RFI, Alstom, AnsaldoBreda, Bombardier, RENFE and ADIF), as well as the most important research groups on railway problems (University of Birmingham, POLIMI, University of Madrid) collaborated to analyse this specific item.

Within both these projects, different experimental campaigns were performed: in field, to characterised the air flow in the underbody zone (Kaltenbach et al., 2008 and Sima et Al., 2011), and in wind tunnel, trying to identify the most important parameters and the thresholds associated to the ballast lifting phenomenon.

In particular, the first experimental campaign was carried out in the SUMKA wind tunnel on 1:10 scale models of the track with the target of defining, for each of them, the mean wind speed threshold when the ballast flying comes up. The results are useful in terms of comparison between different track configurations but not in terms of absolute value due to the simplified operating and boundary conditions. A second experimental campaign was performed in the CSTB wind tunnel (Saussine and Paradot, 2011) on 1:1 scale model. In these tests, the boundary condition due to the train passing is reproduced by a model of the train underbody zone statically set over the ballast, the vibration induced by the vehicle passage is reproduced by moving in vertical direction a sleeper and a gust is reproduced by a sudden opening of a grid.

Moreover, the researchers of the Korea Rail-Road Research Institute conducted tests in wind tunnel to highlight the influence of shape and weight of the stones on the lifting phenomenon but without any modelisation of the infrastructure track (Kwon and Park, 2008). Similar studies, focused on the effect of the shape of the stones and performed by numerical simulations, are described also in Sanz-Andres and Navarro-Medina, 2010 and in Lazaro and Gonzalez, 2011. 
The present paper deals with the study of the ballast lifting phenomenon using wind tunnel tests on a 1:1 track ballast section, with real sleepers, rails and stones. The aim of the research is to investigate the effects on the critical wind speed when the stones begin rolling or flying of the following parameters:

- height of the ballast level with respect to the top of sleeper;

- shape and weight of the stones in the upper layer of ballast;

- compaction of ballast.

First of all, a trackside measurements experimental campaign was performed in order to measure the flow in the underbody region of the vehicle and the accelerations induced by the train passage on the ballast (Giappino et al., 2013). These measurements were adopted to model the experimental conditions during the wind tunnel tests. In particular, specific attention was paid to reproduce the vertical velocity profile, especially close to the ballast level. For this reason, a square cylinder was placed before the test section so that the accelerated flow obtained was comparable with the one measured in the experimental tests on the Italian high-speed line.

Moreover, the ballast was moved according to the measured vertical acceleration by means of a hydraulic actuator.

The tests were performed in the $4 \mathrm{x} 4 \mathrm{~m}$ test section of the Politecnico di Milano wind tunnel, whose maximum wind speed is $55 \mathrm{~m} / \mathrm{s}$.

Starting from the results of these tests, possible countermeasures to ballast lifting on-set are proposed in the conclusions.

\section{TRACKSIDE EXPERIMENTAL TESTS}

In order to characterize the flow in the underbody region and the dynamics of the whole track (rail, sleepers and ballast), several experimental campaigns were carried out on the Italian high-speed network on the lines Milan-Turin (Alice, Recetto and Greggio) and Rome-Naples (Cassino). The objective was to measure both the aerodynamic variables (air pressure, velocity profile over ballast and aerodynamic loads on stones) and the mechanical vibrations of the railway infrastructure due to the train passing. In this paper only the main results, useful for the design of the wind tunnel test campaign, will be shown; the complete data analysis of these field experimental campaigns is presented in Giappino et al., 2013. 


$$
C_{u}(z)=\frac{U(z)}{V_{\text {train }}}
$$


In Figure 3 the wind speed measured by the upper pitot tube of the vertical array for two type of trains (whose characteristics are summarised in Table 1) is shown. These results were

121 obtained averaging the time histories of several passages (about 30 for the first train and 10 for

122 the second one) so that the non-correlated contributions were eliminated. It is possible to notice

123 a first peak of speed (corresponding to the overpressure in front of the train head at $\mathrm{x}=0$ ); then

124 the speed stabilises with periodic oscillations due to the passage of the coaches and finally a

125 second peak occurs when the last car of the train passes over the instrumented section. The 126 oscillations are strongly influenced by the shape of the underbody region and by the numbers 127 of bogies; with the first type of train (13 cars) ) the "steady state" is quickly reached after two 128 coaches while with the second type it is reached after five coaches and the oscillations ampli129 tude is lower. These differences could be explained observing the characteristics of the two 130 trains showed in Table 1; in the first case, the nose is shorter and there are more bogies, ele131 ments that surely contribute to increase the flow speed. To be thorough, it must be underlined 132 that in the second case less passages were averaged, with the result of a time history more noisy 133 and non-correlated.

134 As regard to the harmonic content of the signals, as previously done, in order to make the results 135 independent from the train speed, it is possible to present the data as a function of the wave136 length:

$$
\lambda=\frac{V_{\text {train }}}{f}
$$

\begin{tabular}{|c|c|c|}
\hline & 13 cars train & 11 cars train \\
\hline TOTAL LENGTH & $330 \mathrm{~m}$ & $200 \mathrm{~m}$ \\
\hline NOSE LENGTH & $4 \mathrm{~m}$ & $6 \mathrm{~m}$ \\
\hline NUMBER OF CARS & 13 & 11 \\
\hline NUMBERS OF BOGIES & 26 & 12 \\
\hline TRACTION & concentrated & distributed \\
\hline
\end{tabular}


141 The result is that the periodic oscillation at low-frequency is associated to the coach length (26

$142 \mathrm{~m})$. Considering a train speed of $300 \mathrm{~km} / \mathrm{h}$, the corresponding frequency lies around 3-4 Hz.

143 Averaging the values of the $c_{u}$ distribution along the train on the central portion, it is possible

144 to calculate a mean vertical profile of the flow velocity longitudinal component. In Figure 4 the

145 results obtained in different experimental campaigns with two types of trains are compared. All

146 the curves show a similar slope but different absolute values; this might be due to different

147 height of the ballast and different underbody regions (smoother for the 11 cars train). In partic-

148 ular, the lower point set under the top of the sleeper, is characterized by an almost null velocity.

149 Finally, the averaged value of all the three components of the flow velocity and forces are 150 showed in Figure 5 in the case of 13 cars train.

Figure 4 Vertical speed profiles measured with the pitot tubes array

Figure 5 Time histories of the velocity components of the flow (a) and the three components of the aerodynamic force acting on the cube (b)

\subsubsection{Accelerations}

158

159

160

161

162

163

164

165

166

167

168

169

Aim of measuring accelerations of stones and aerodynamic forces acting on them is to characterise the ballast conditions (aerodynamic forces and dynamics) to understand which are the key parameters in the flying ballast phenomenon. In particular, many authors agree that the initial part of the ballast projection phenomenon is associated with a phase of rolling and this is aided by initial upward velocity ([citazione]). Moreover, also downward accelerations, and the consequent inertia forces which reduce the total vertical forces (which means less friction with particles below), facilitate rolling.

In order to measure and to chart the level of the accelerations of the entire track, several accelerometers were placed over rails, sleepers and stones. The accelerometers used, all uniaxial, were set as described in Figure 2. In particular, two of the six accelerometers connected to the stones were put about $10 \mathrm{~cm}$ under the upper layer of the ballast, in order to highlight possible differences as a function of the depth.

All the signals are low pass filtered at $40 \mathrm{~Hz}$ in order to remove all the harmonic components at high frequency that cannot excite the dynamics of the stone. The vertical acceleration (Figure $6 a)$ is characterised by a periodic behaviour associated to the passage of the axles. It is important to notice that the maximum acceleration of the ballast, as expected, does not exceed one $\mathrm{g}$. 
173 Furthermore, all the stones are nearly exposed to the same level of vibration (Figure 6b): this

174 means that the entire ballast vibrate with the same amplitude independently from the position.

175

176

177

178

179

180

181

182

183

184

185

186

187

188

189

190

191

192

193

194

195

196

197

198

199

200

201

202

Figure 6 Time evolution of the vertical acceleration of a stone in the case of 13 cars train (a) and comparison between the accelerations measured on three different ballast stones (b)

On the other hand, the acceleration is linked to the weight of the single axle and to the speed of the train. In the case of trains with 13 cars, where the weight is concentrate in the locomotive, the accelerations reached $0.3 \mathrm{~g}$ while for the other coaches are rather lower. This means that, during the passage of the axles, the weight force of the single stone and, consequently, the friction with the lower layers reduces of $30 \%$.

\section{WIND TUNNEL EXPERIMENTAL TESTS}

Starting from the results obtained from the trackside measurements, experimental tests on a full-scale track section were designed and carried out in the wind tunnel of the Politecnico di Milano. The experimental campaign was carried out in the $4 \mathrm{~m} \times 4 \mathrm{~m}$ test chamber where a maximum wind speed of $55 \mathrm{~m} / \mathrm{s}$ can be reached.

A full-scale model 4 meters long was used. The model consists of a track section of a real Italian high-speed railways with ballast sleepers and rails (Figure 9). The main objective of the test was to identify, in a controlled site, critical speeds, intended as feeding speed of the train, at which there is the incipient lifting of the ballast stones with the possibility to change various test conditions of the track such as ballast height, vibration level and compaction level.

\subsection{Experimental set-up}

A four meters long stretch of the Italian high-speed railways has been rebuilt into the Politecnico di Milano wind tunnel section.

The test section size allowed to place into the test room seven sleepers (60 centimeters spaced). To decrease the initial discontinuity, an aerodynamic profile was placed ahead of the first sleeper to avoid vortex shedding from its edge (Figure 8).

The target of the wind tunnel test is to reproduce the real conditions over the track when the train is passing in terms of:

- mean flow speed vertical profile over the ballast surface;

- vertical track vibration due to the wheel-rail contact. 
The mean wind speed vertical profile was obtained by simulating a track model long enough upwind the inter-sleepers gap selected as the test section. In addition, a square cylinder was positioned above the fourth sleeper in order to increase the wind speed over the ballast (Figure 7a). Nevertheless, the cylinder, besides accelerating the flow, introduces also a certain level of turbulence due to vortex shedding. Unfortunately, it was not possible to reproduce the real frequency. In fact, considering a train speed of $300-330 \mathrm{~km} / \mathrm{h}$, the frequency of the flow speed fluctuations is about 3.3-3.7 Hz. Assuming a Strouhal number of $\mathrm{St}=0.12$ for a cylinder with a square section, from the Strouhal relation between the wind speed $V\left(V_{\max }=50 \mathrm{~m} / \mathrm{s}\right)$ and the desired frequency f:

$$
S t=\frac{f L}{V}
$$

the edge of the square should be 1.8-2 meter long. A profile of such dimensions is naturally impossible to use in a tunnel section of $4 \times 4$ meters.

However, a smaller cylinder with a side of $40 \mathrm{~cm}$ was placed over the fourth sleeper (Figure 9) in order to have the opportunity to carry out tests with an equivalent speed of the train up to $400 \mathrm{~km} / \mathrm{h}$. The frequency of vortex shedding with this cylinder is higher $(10-15 \mathrm{~Hz}$ as shown in Figure 7b) and the amplitude of the oscillations is equivalent to that of the averaged timehistory measured through field tests but it is obviously lower than that of the single passage. At any rate, it is authors' opinion that, for a sensitivity analysis of the parameters that influence the ballast lifting, it is more important to correctly reproduce the mean flow characteristics; fluctuations, in fact, may increase the number of events, but these would not change the results obtained in terms of qualitative analysis.

Figure 7 Comparison between the wind speed measured in the wind tunnel with and without the cylinder (a) and an example of flow obtained (b)

Figure 8 Full-scale model in the wind tunnel test section Figure 9 Representation of the experimental set-up inside the wind tunnel

The vertical accelerations of the ballast were imposed only in correspondence of the fourth inter-sleepers gap using a hydraulic actuator (Figure 10). The input of the actuator control system were the accelerations measured on the ballast (2.1.3), in order to obtain a vibration of the upper layer of the ballast similar to that measured trackside. Since the accelerations measured were independent from the position of the stones on the track it was possible to simplify the problem and move all the ballast together. 


\subsubsection{Measurements system}

The measurements were carried out in two steps:

- in the first one the flow and the acceleration of the ballast generated in the wind tunnel

\subsubsection{Measurements of flow and acceleration}

Regarding the measure of the flow field the same instrumentation used trackside (Figure 11) was adopted:

- an array of five pitot tubes;

- a multi-hole probe;

- a cubic pressure transducer;

- three uniaxial accelerometers fixed on the stones.

Moreover, to obtain a complete mapping of the flow velocity in correspondence of the test section a semi-automatic device (called "Traversing") was used. As shown in Figure 12a, the device consists of a mast whose base is fixed to a horizontal actutated guide which allows the movement along the longitudinal axis of the tunnel (x-axis). The mast is equipped with a second motor drive at which is bound an horizontal arm, which can move vertically (z-axis). Finally, at the end of the arm, a vertical rod is fixed laying a multihole probe, as shown in Figure 12b.

\subsubsection{Sensitivity analysis}

In order to identify and record the movements of the ballast stones, the entire test campaign was filmed using a high frame rate camera (300 fps). In this way the stone behavior in the whole test section was controlled (Figure 13) and a detailed classification of the different types of occurred events were possible.

Figure 11 Pitot tubes array (a) and accelerometers over the ballast (b) 
The first part of the experimental campaign has focused on the characterization of the conditions of the flow in the wind tunnel. In particular, we tried to find out the best position of the cylinder in order to recreate the same conditions as measured trackside. Due to the high variability of the data presented in 2.1.2 some simplifications were made:

- only the average flow was taken into account without fluctuations and peaks;

- the target profile was an average of the ones measured trackside without considering the differences due to different type of trains.

\subsubsection{Vertical profile of mean wind speed}

In order to compare the vertical profile of the flow mean speed reproduced in the wind tunnel with the one measured at trackside, a comparison is presented in a dimensionless form accord272 ing to the following expression:

$$
C_{u}(z)=\frac{U(z)}{V_{\text {train_wt }}}
$$

274 where $\mathrm{U}(\mathrm{z})$ represents the average value of the speed at a certain height $\mathrm{z}$ from the top of rail 275 (TOR) and $V_{\text {train_wt }}$ is the equivalent speed of the train. Neglecting Reynold-dependent effects 276 it is possible to measure the profiles in the wind tunnel at a nominal speed test of $25 \mathrm{~m} / \mathrm{s}$ (rather 277 limited in order to avoid a possible lifting of the ballast that could damage the measuring set) 278 and report the dimensionless results to higher speeds. Assuming as reference the wind speed 279 (called as $U_{r e f_{-} w t}$ in the wind tunnel and $U_{\text {ref_re }}$ in the trackside situation) measured by the 280 highest pitot (27 mm below the TOR), it is possible to calculate the ratio between the nominal 281 speed of the wind tunnel $\left(U_{w t}\right)$ and the equivalent speed of the train $\left(U_{\text {train_wt }}\right)$ as:

$$
C=\frac{U_{w t}}{V_{\text {train_wt }}}=\frac{U_{w t}}{U_{\text {ref_wt }}} \cdot \frac{U_{\text {ref_wt }}}{V_{\text {train_wt }}}=\frac{U_{w t}}{U_{\text {ref_wt }}} \cdot \frac{U_{\text {ref_re }}}{V_{\text {train_re }}}=0.872 \cdot 0.49=0.427
$$

282 That means:

$$
V_{\text {train_eq }}=1 / C \cdot U_{w t}=C_{e q} \cdot U_{w t}=2.34 \cdot U_{w t}
$$


284 It is important to underline that the ratio between the nominal speed of the wind tunnel and the 285 wind speed of the reference pitot is lower than one (0.872). This means that, as already said in 286 3.1, the cylinder over the fourth sleeper, coupled with the effect of blockage of the model, 287 accelerate the flow in the test section. In Figure 7a the profiles measured with the array of pitot 288 tubes in the wind tunnel, with and without the cylinder, are compared while in Figure $7 \mathrm{~b}$ the 289 profile obtained with the cylinder is compared with the ones measured trackside.

Figure 14 Comparison between the vertical profile measured trackside with the one obtained in the wind tunnel

292 From this comparison, it is possible to observe that the agreement between the profiles is very 293 good up to a height of about $150 \mathrm{~mm}$ below the TOR especially with regard to the slope; under 294 this limit the profile has an evident deviation. The lower point is the closer one to the ballast and, consequently, it is also the most critical measurement: in this zone (under the plane of the sleepers) the flow is strongly influenced by the superficial disposition of the stones and it is characterized by high gradient and turbulence. In order to better define this zone, the "Traversing" was used and several speed profile were measured in different locations (Figure 15a). The results, shown in Figure 15b, demonstrate the goodness of the flow field obtained in the wind tunnel, compared to the one measured on track, and the high gradient of the speed starting from $190 \mathrm{~mm}$ below the TOR (that means $20-30 \mathrm{~mm}$ above the sleepers). In particular the profiles in the same inter-sleepers gap of the pitot tubes array are almost the same while the ones measured in the next inter-sleepers gap show lower speed; this is due to the acceleration effect of the cylinder.

Figure 15 Different vertical profile measured with the Traversing (b) with ballast at $\mathbf{- 3} \mathbf{c m}$ and their positions (a)

307 To be thorough, a comparison of profiles with two different levels of the ballast is reported in 308 Figure 16. Lowering the level of the ballast the speed increase particularly near the sleepers due to the reduced equivalent superficial roughness. On the other hand, the upper layer of the ballast is located in a lower position; although the wind speed at the level of the plane of the sleepers is higher, it is lower at the level of the ballast. 
Regarding the pressures measured with the multi-hole cube, in Figure 17 a comparison of three different situation is showed. The first and second pictures represent the trackside measurements with the cube placed at two different levels: $20 \mathrm{~mm}$ above the sleepers (a) and at the same level of the plane of the sleepers (b). The third image, however, refers to the tests made in the wind tunnel where the cube was at the same level of (b). The comparison between (b) and (c) shows that in the wind tunnel has been achieved a good correspondence in respect to the trackside campaigns also from the point of view of the pressures. On the other hand, in this area was not possible to compare the profiles obtained in the wind tunnel with the ones of the pitot array. In order to obtain a comparison for the profiles, we can suppose that the central section of the cube is a stagnation point; in this way it is possible to compute the speed at this level and compare it with the profile made with the "Traversing" (Figure 18). The results demonstrate that the slope of the speed profile significantly change 10-20 $\mathrm{mm}$ above the sleepers and the speed quickly decreases also in the inter-sleepers gap. The same result is obtained also integrating the forces acting on the cube as showed in Table 2.

Figure 17 Comparison between the pressures measured trackside at Recetto (a) and Greggio (b) and in the wind tunnel (c)

Figure 18 Vertical profile measured in the wind tunnel compared with the speed seen by the cube

\begin{tabular}{|c|c|c|c|c|}
\hline & $\begin{array}{c}\text { ALICE } \\
\boldsymbol{h}_{\text {cube }}=\mathbf{2 0 m m}\end{array}$ & $\begin{array}{c}\text { RECETTO } \\
\boldsymbol{h}_{\text {cube }}=\mathbf{2 0 m m}\end{array}$ & $\begin{array}{c}\text { GREGGIO } \\
\boldsymbol{h}_{\text {cube }}=\mathbf{0 m m}\end{array}$ & $\begin{array}{c}\boldsymbol{W T} \\
\boldsymbol{h}_{\text {cube }}=\mathbf{0 m m}\end{array}$ \\
\hline $\mathrm{C}_{\mathrm{u}}[-]$ & 0.52 & 0.50 & 0.49 & 0.48 \\
\hline $\mathrm{C}_{\mathrm{v}}[-]$ & 0.00 & -0.01 & 0.00 & 0.00 \\
\hline $\mathrm{C}_{\mathrm{w}}[-]$ & -0.02 & -0.04 & -0.03 & 0.01 \\
\hline $\mathrm{F}_{\mathrm{x}}[\mathrm{N}]$ & 1.45 & 1.49 & 1.09 & 1.14 \\
\hline $\mathrm{F}_{\mathrm{y}}[\mathrm{N}]$ & 0.00 & 0.00 & 0.00 & 0.00 \\
\hline $\mathrm{F}_{\mathrm{z}}[\mathrm{N}]$ & -0.89 & -0.78 & -0.65 & -0.62 \\
\hline
\end{tabular}

Table 2 Comparison between velocity components and aerodynamic forces trackside and in the wind

\subsubsection{Acceleration of ballast} tunnel

In Figure 19 accelerations measured in the two situations, trackside and in the wind tunnel, are showed; a good correspondence was obtained. 


\subsection{Test typology}

339 Once the flow has been characterized, the instrumentation was removed and the experi340 mental campaign was performed with increasing speeds, corresponding to increments of $10 \%$ 341 of the power of the wind tunnel, starting from $50 \%$ and reaching up to $100 \%$ (Table 3 ).

\begin{tabular}{|c|c|c|}
\hline WT power [\%] & Nominal speed [m/s] & Equivalent speed [km/h] \\
\hline 50 & 25 & 217 \\
\hline 60 & 30 & 260 \\
\hline 70 & 35 & 304 \\
\hline 80 & 40 & 347 \\
\hline 90 & 45 & 390 \\
\hline 100 & 50 & 434 \\
\hline
\end{tabular}

Table 3 Correspondences between wind tunnel power and equivalent train speed

344 The single test at a given speed was comprised of two parts: once the full speed of the test was 345 reached it was maintained for about two minutes without vibrations; subsequently the actuator 346 was turned on monitoring the behavior of the system for others two minutes.

347 In order to investigate the effects, on the ballast lifting, of different track conditions and to 348 seek the most effective countermeasures that can be adopted, the tests were repeated by chang349 ing the following parameters:

- height of ballast in respect to the upper surface of the sleepers (Figure 20a);

- $\quad$ presence of stones of different shape in the surface layer (Figure 20b);;

353 These parameters can be combined getting different combinations as shown in Table 4.

\begin{tabular}{|c|c|c|c|}
\hline Test ID & Ballast height & Stones shape & Compaction \\
\hline P0R_- & $\mathrm{Z}_{b}=0 \mathrm{~cm}$ & Random & No \\
\hline P0RC & $\mathrm{Z}_{b}=0 \mathrm{~cm}$ & Random & Yes \\
\hline P0F_ & $\mathrm{Z}_{b}=0 \mathrm{~cm}$ & Flattened & No \\
\hline P3R_- & $\mathrm{z}_{b}=-3 \mathrm{~cm}$ & Random & No \\
\hline P3RC & $\mathrm{z}_{b}=-3 \mathrm{~cm}$ & Random & Yes \\
\hline P3F_- & $\mathrm{z}_{b}=-3 \mathrm{~cm}$ & Flattened & No \\
\hline
\end{tabular}




\begin{tabular}{|l|l|l|l|}
\hline P5R_- & $\mathrm{z}_{\mathrm{b}}=-3 \mathrm{~cm}$ & Random & No \\
\hline P5F_- & $\mathrm{z}_{\mathrm{b}}=-5 \mathrm{~cm}$ & Flattened & No \\
\hline
\end{tabular}

Table 4 List of tests carried out with different ballast conditions

355 For each of the three levels of ballast three changes in the conditions of the ballast were made.

356 In the first, stones of different geometry and weight were randomly placed in the upper layer.

357 Subsequently the ballast was compacted moving the actuator at high frequency and compress358 ing the ballast applying a vertical load on it (The compaction was not performed with the ballast $3595 \mathrm{~cm}$ under the sleepers since, due to the stability problems, this situation is not reachable on 360 the railway. Therefore the tests carried out in this situation, have a pure theoretical validity). In 361 the third session stones with flattened shape were placed on the surface (Figure 20b). The aer362 odynamically-favorable shape of these stones (high surface over weight ratio) has been identi363 fied as one of the main parameters linked to the ballast lifting problem.

Figure 20 Examples of ballast conditions: $3 \mathrm{~cm}$ under the sleeper (a) and stones with flattened shape

\subsection{Results}

The events observed during the whole experimental campaign in the wind tunnel were classified defining four different classes, each corresponding to a movement of the stones more or less significant.

In particular, they were defined as:

Class A - no movement caused by the air flow;

Class B - local fluctuations: at least one stone starts to oscillate locally due to the flow turbulence but no displacements are recorded at the end of the test;

Class C - local rolling: at least one stone shows a significant displacement and rolls to another location;

Class D - relevant rolling: at least one stone has been pushed out of the test chamber or passed over a sleeper.

The class is assigned basing on the highest-class event that occurs during a single test: if, for example, a local rolling and a relevant rolling occur together, the entire test is assigned to the 4th class.

\begin{tabular}{|c|c|c|c|c|c|c|}
\hline Test ID & $\mathbf{2 1 7} \mathbf{~ k m} / \mathbf{h}$ & $\mathbf{2 6 0} \mathbf{~ k m} / \mathbf{h}$ & $\mathbf{3 0 4} \mathbf{~ k m} / \mathbf{h}$ & $\mathbf{3 4 7} \mathbf{~ k m} / \mathbf{h}$ & $\mathbf{3 9 0} \mathbf{~ k m} / \mathbf{h}$ & $\mathbf{4 3 0} \mathbf{~ k m} / \mathbf{h}$ \\
\hline P0R_- & A & A & AABC & ABCDD & CCC & \\
\hline P0RC & & & & & B & A \\
\hline
\end{tabular}




\begin{tabular}{|c|l|l|l|c|c|c|}
\hline P0F_ & & & & CCCDD & D & \\
\hline P3R_ & & & & B & B & \\
\hline P3RC & & & & & AC & AB \\
\hline P3F_ & & & & BBCD & CDD & \\
\hline P5R_- & & & A & A & B \\
\hline P5F_ & & & & A & B & B \\
\hline
\end{tabular}

Table 5 Results without vibration

\begin{tabular}{|c|c|c|c|c|c|c|}
\hline Test ID & $\mathbf{2 1 7} \mathbf{~ k m} / \mathbf{h}$ & $\mathbf{2 6 0} \mathbf{~ k m} / \mathbf{h}$ & $\mathbf{3 0 4} \mathbf{~ k m} / \mathbf{h}$ & $\mathbf{3 4 7} \mathbf{~ k m} / \mathbf{h}$ & $\mathbf{3 9 0} \mathbf{~ k m} / \mathbf{h}$ & $\mathbf{4 3 0} \mathbf{~ k m} / \mathbf{h}$ \\
\hline P0R_ & A & A & AAABC & AADD & CD & \\
\hline P0RC & & & & & B & A \\
\hline P0F_ & & & & CCCCD & D & \\
\hline P3R_ & & & & A & AB & \\
\hline P3RC & & & & & AC & AB \\
\hline P3F_ & & & & ABBC & CDD & \\
\hline P5R_ & & & & A & A & B \\
\hline P5F_ & & & & A & B & B \\
\hline
\end{tabular}

Table 6 Results with vibration

383 In Table 5 and Table 6 the classes assigned at each repetition of each test at different equivalent 384 train speed are reported. Looking at Table 5, starting from the Italian railway situation, i.e. 385 ballast at the same level of the sleepers $(\mathrm{Z}=0 \mathrm{~cm})$ with random arrangement of stones (P0R), a 386 critical velocity is not evident. This is due to the randomness of this phenomenon (linked to the 387 random arrangement of the stones) and to the small statistical sample (few sleepers and few 388 repetition). On the other hand, in presence of flat and light stones on the surface (P0F) all the 389 trials showed the higher classes.

390 Continuing the analysis of the results reported in the table, in the case of ballast lowered by 3 $391 \mathrm{~cm}(\mathrm{P} 3)$ a reduction of the assigned classes during tests with random arrangement of stones 392 (P3R) is observed. However, the placement of flat stones again shows important events at 393 speeds comparable with the previous case.

394 Finally lowering the ballast level $5 \mathrm{~cm}$ below the sleepers presents a marked improvement, 395 resulting in the allocation of only classes A and B in all cases. 
The compaction of the ballast definitely seems to have a beneficial effect to all levels of the ballast while the comparison between Table 5 and Table 6 (that means with and without vibrations) does not show substantial differences.

On the other hand, the real situation is different and pejorative since the maximum acceleration and the gust of wind acting in phase, because they are both generated by the passages of the bogies, in the wind tunnel, instead, vortex shedding is not correlated with the vibrations.

\section{CONCLUSIONS}

An experimental campaign in the Politecnico di Milano wind tunnel was carried out with the aim to identify the most important parameters that have a role in the phenomenon of flying ballast and possible counter-measures to adopt.

Referring to the overall results obtained in different tests we can assert that:

- the flow over the track is characterized by an high gradient starting from $2-3 \mathrm{~cm}$ above the sleepers where there is a clear change in the slope of the vertical profile;

- lowering the level of the ballast under the plane of the sleepers has undoubtedly a positive effect; despite the condition with ballast lowered by $5 \mathrm{~cm}$ is not practicable, a decrease of $3 \mathrm{~cm}$ brings to great benefits; due to the high gradient of the speed profile, although the wind speed at the level of the sleepers is higher, it is lower at the level of the ballast;

- the shape of the stones affect the phenomenon; in particular, the presence of flat stones on the surface increases the likelihood to lifting. These stones are light but with high kinetic energy which could be transferred to other heavier stones;

- the vibration does not appear to have a significant role in the phenomenon.

In conclusion, it is important to underline that the mean flow characteristics obtained in the tunnel were very similar to those measured trackside, both from the point of view of speed profile and pressures. As far as the dynamic properties of the flow are concerned, the wind speed oscillations reproduced in the wind tunnel are characterized by a higher frequency and a lower amplitude (especially compared to the single passage) than those measured through real scale tests. Furthermore, in the real conditions, the oscillations are synchronous with the vertical accelerations. That means that all the results obtained in the wind tunnel are probably less conservative with respect to the real situation. On the other hand, the final goal of the paper is to perform a sensitivity analysis and not to evaluate a wind speed threshold over that the ballast flies, also considering the reduced statistical basis of a stretch only four meters long. Differences in oscillations can modify the number of events or change the values of the threshold 
429 speed, but they do not influence the performed qualitative analysis whose final target is to un430 derline the parameters, from the point of view of the railway, that can be controlled to reduce 431 the ballast lifting. 


\section{REFERENCES}

433 H.B. Kwon, C. S. Park, An Experimental Study on the Relationship between Ballast-flying Phe434 nomenon and Strong Wind under High-speed Train.

435 Proceeding of the World Congress on Rail Research, 2008.

D. Quinn, M. Hayward, C. J. Baker, F. Schmid, J. A. Priest, W. Powrie, A full-scale experi438 mental and modelling study of ballast flight under high-speed trains.

439 Proceedings of the Institution of Mechanical Engineers, Part F: Journal of Rail and Rapid 440 Transit, 2010.

441

442 A. Sanz-Andres, F. Navarro-Medina, The initiation of rotational motion of a lying object 443 caused by wind gusts.

444 Journal of wind Engineering and Industrial Aerodynamics, 98, 2010.

446 G. Saussine, E. Allain, N. Paradot, V. Gaillot, Ballast Flying Risk Assessment Method for High 447 Speed Line.

448 9th World Conference on Railway Research , 2011.

449

450 S. Giappino, S., Premoli, A., Rocchi, D., Schito, P., Tomasini, G., 2013, Numerical-experi451 mental study on flying ballast caused by high speed trains

452 EACWE, 6th European and African Conference on Wind Engineering, Cambridge, July 7thto 453 11th 2013

454

455

B. J. Lazaro, E. Gonzalez, - Characterization and Modelling of Flying Ballast Phenomena in 456 High-speed train lines.

457 9th World Conference on Railway Research , 2011.

459 Jing, G.Q, Zhou, Y.D, Lin, J., Zhang, J. Ballast flying mechanism and sensitivity factors anal$460 \quad y$ sis

461 International Journal on Smart Sensing and Intelligent Systems, 5 (4), 928-939, 2012 462

463 M. Sima, E. Grappein, M. Weise, N. Paradot, M. Hieke, C. Baker, R. Licciardello, M. Couturier 464 Presentation of the EU FP7 AeroTRAIN project and first results 
465 9th World Conference on Railway Research , 2011.

466

467 H.J. Kaltenbach, P. E. Gautier, G. Agirre, A. Orellano, K. Schroeder, Bodenstein, M. Testa,

468 Th. Assessment of the aerodynamic loads on the trackbed causing ballast projection:

469 results from the DEUFRAKO project Aerodynamics in Open Air (AOA)

470 8th World Conference on Railway Research , 2008 
FIGURES

472

473 Figure 1 Experimental set-up for the flow measurements

474

475 Figure 2 Layout of the accelerometers on sleepers and ballast

476

477 Figure 3 Averaged time histories of the upper pitot tube for the 13 cars train (a) and the 11 cars 478 train (b)

479

480

Figure 4 Vertical speed profiles measured with the pitot tubes array

481

Figure 5 Time histories of the velocity components of the flow (a) and the three components of 483 the aerodynamic force acting on the cube (b)

484

Figure 6 Time evolution of the vertical acceleration of a stone in the case of 13 cars train (a) and comparison between the accelerations measured on three different ballast stones (b)

Figure 7 Comparison between the wind speed measured in the wind tunnel with and without the cylinder (a) and an example of flow obtained (b)

Figure 8 Full-scale model in the wind tunnel test section

Figure 9 Representation of the experimental set-up inside the wind tunnel

Figure 10 Particular of the hydraulic actuator under the fourth inter-sleepers gap

Figure 11 Pitot tubes array (a) and accelerometers over the ballast (b)

Figure 12 "Traversing" inside the wind tunnel (a) and a particular of the multi-hole probe (b)

Figure 13 Position (a) and example of the view of the camera (b)

503 Figure 14 Comparison between the vertical profiles measured trackside with the one obtained 504 in the wind tunnel 
506 Figure 15 Different vertical profile measured with the Traversing (b) with ballast at $-3 \mathrm{~cm}$ and 507 their positions (a)

508

509 Figure 16 Comparison of the vertical profiles with different levels of ballast

510

511 Figure 17 Comparison between the pressures measured trackside at Recetto (a) and Greggio

512 (b) and in the wind tunnel (c)

513

514 Figure 18 Vertical profile measured in the wind tunnel compared with the speeds seen by the 515 cube

516

517 Figure 19 Acceleration measured trackside (a) and in the wind tunnel (b)

518

519 Figure 20 Examples of ballast conditions: $3 \mathrm{~cm}$ under the sleeper (a) and stones with flattened 520 shape (b) 